\title{
Beam Dynamics of Muon Acceleration for Neutrino Factory*
}

\author{
S.A. Bogacz ${ }^{\star}$ Jefferson Lab, Newport News, VA 23606, USA
}

\begin{abstract}
A conceptual design of a muon acceleration scheme based on recirculating superconducting linacs is proposed. In the presented scenario, acceleration starts after ionization cooling at $245 \mathrm{MeV} / \mathrm{c}$ and proceeds to $20 \mathrm{GeV}$, where the beam is injected into a neutrino factory storage ring. The key technical issues are addressed; such as: the choice of acceleration technology (superconducting versus normal conducting) and the choice of RF frequency, and finally, implementation of the overall acceleration scheme: capture, acceleration, transport and preservation of large phase space of fast decaying species. Beam transport issues for large-momentum-spread beams are accommodated by appropriate lattice design choices. The proposed arc optics is further optimized with a sextupole correction to suppress chromatic effects contributing to emittance dilution.
\end{abstract}

\section{Muon Acceleration Scheme}

A neutrino factory [1] is aimed to produce narrow neutrino beams via decay of muons in long straight sections of a storage ring. As illustrated schematically in Figure 1, a proposed muon accelerator complex features a 0.2 -to- $2.8 \mathrm{GeV}$ straight pre-accelerator linac and a 2.8 -to- $20 \mathrm{GeV}$ four-pass recirculating linac accelerator (RLA).

The pre-accelerator captures a large muon phase space coming from the cooling channel and accelerates them to relativistic energies of about $2.8 \mathrm{GeV}$. It makes the beam sufficiently relativistic and adiabatically decreases the phase-space volume, so that effective acceleration in recirculating linacs is possible. The RLA further compresses and shapes the longitudinal and transverse phase spaces, while increasing the energy.

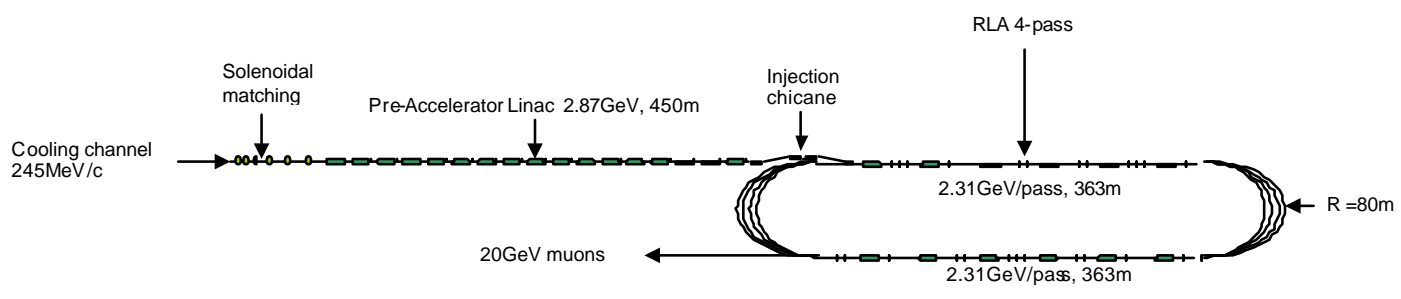

Figure $120 \mathrm{GeV}$ muon accelerator complex based on RLA - machine layout.

\section{Accelerating Technology - Design Choices}

To ensure adequate survival rates of short-lived muons, acceleration must occur at high average gradient. Initial estimate [2] shows that a "real estate average" RF gradient of $15 \mathrm{MV} / \mathrm{m}$ will allow survival of about $80 \%$ of source muons throughout the RLA. Since muons are generated as a secondary beam they occupy large phase-space volume. The accelerator must provide high average gradient, while maintaining very large transverse and longitudinal accelerator acceptances. The above requirement drives the design to low RF frequency, e.g. $200 \mathrm{MHz}$. If normalconducting cavities at that frequency were

\footnotetext{
* Work supported by the US DOE under contract \#DE-AC05-84ER40150

- e-mail: bogacz@jlab.org
} 
used, the required high gradients would demand unachievably high peak RF sources. The RF power can then be delivered to the cavities over an extended time, and thus RF source peak power can be reduced.

\section{Machine Architecture}

In a recirculating linac accelerator one needs to separate different energy beams coming out of a linac and to direct them into appropriate arcs for recirculation.

For multiple practical reasons horizontal rather than vertical beam separation was chosen [2]. Rather than suppressing horizontal dispersion created by the spreaders and recombiners it is smoothly matched to the horizontal dispersion of the arc. Finally, to assure compact arc architecture very short matching sections in spreaders and recombiners are desired (see Figure 2).

One also needs to maintain manageable beam sizes in the arcs [3]. This calls for short cells and for putting stringent limits on dispersion and beta functions (beam envelope). Since spreaders and recombiners were chosen in the horizontal plane, the uniform focusing and lattice regularity was broken in that plane and the horizontal beam envelope requires special attention. On the other hand, the vertical beam size remains small due to maintaining uniform focusing and small beta functions in that plane.
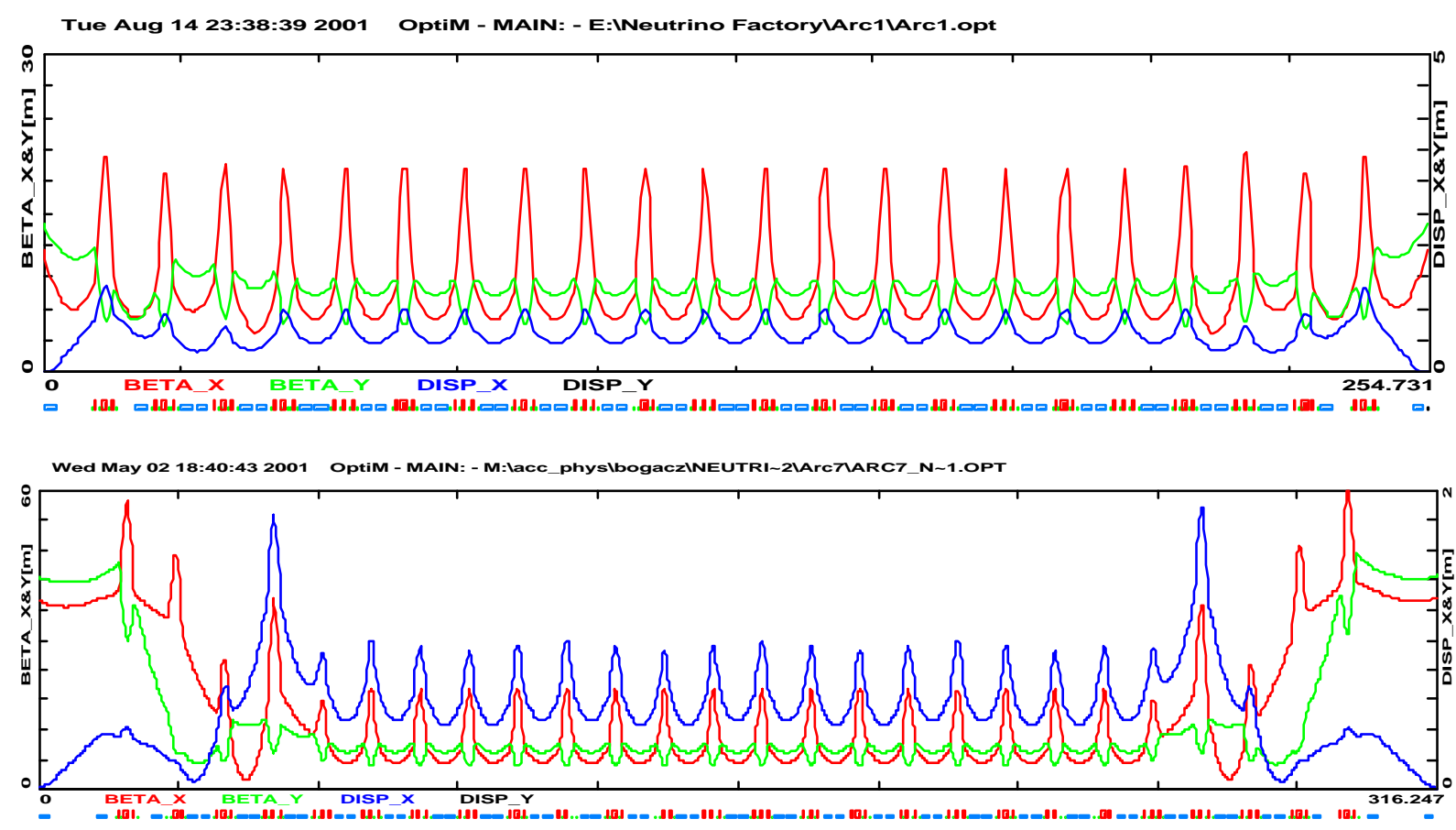

Figure 2 Optics of Arc1 (top) and Arc7 (bottom) - beta-functions and the horizontal dispersion matched to both adjacent linacs; a quest to maintain 'smooth' transition of beta functions across spreaders and recombiners.

\section{Longitudinal Bunch Compression}

Bunch length and energy spread are still too large at the RLA input and their further compression is required in the course of acceleration. To achieve this the beam is accelerated off-crest with non zero $\mathrm{M}_{56}$. That causes synchrotron motion, which suppresses the 
longitudinal emittance growth related to non-linearity of accelerating voltage. Without synchrotron motion the minimum beam energy sread would be determined by nonlinearity of RF voltage at bunch length and would be equal to $(1-\cos \phi) \approx 6 \%$ for bunch length $\phi=20 \mathrm{deg}$. The synchrotron motion causes particle motion within the bunch and averages the total energy gain of tail's particle to the energy gain of particles in the core. The optimum value is about $1.4 \mathrm{~m}$, while optimal detuning of RF phase from on-crest position is different for different arcs. As one can see although longitudinal motion is still quite non-linear it is possible to reduce the energy spread by 4.7 times to $\pm 1.6 \%$ with emittance dilution of about $75 \%$. Final details of beam dynamics depend on the beam transport optics and can be only determined by longitudinal phase-space tracking [2] illustrated below:
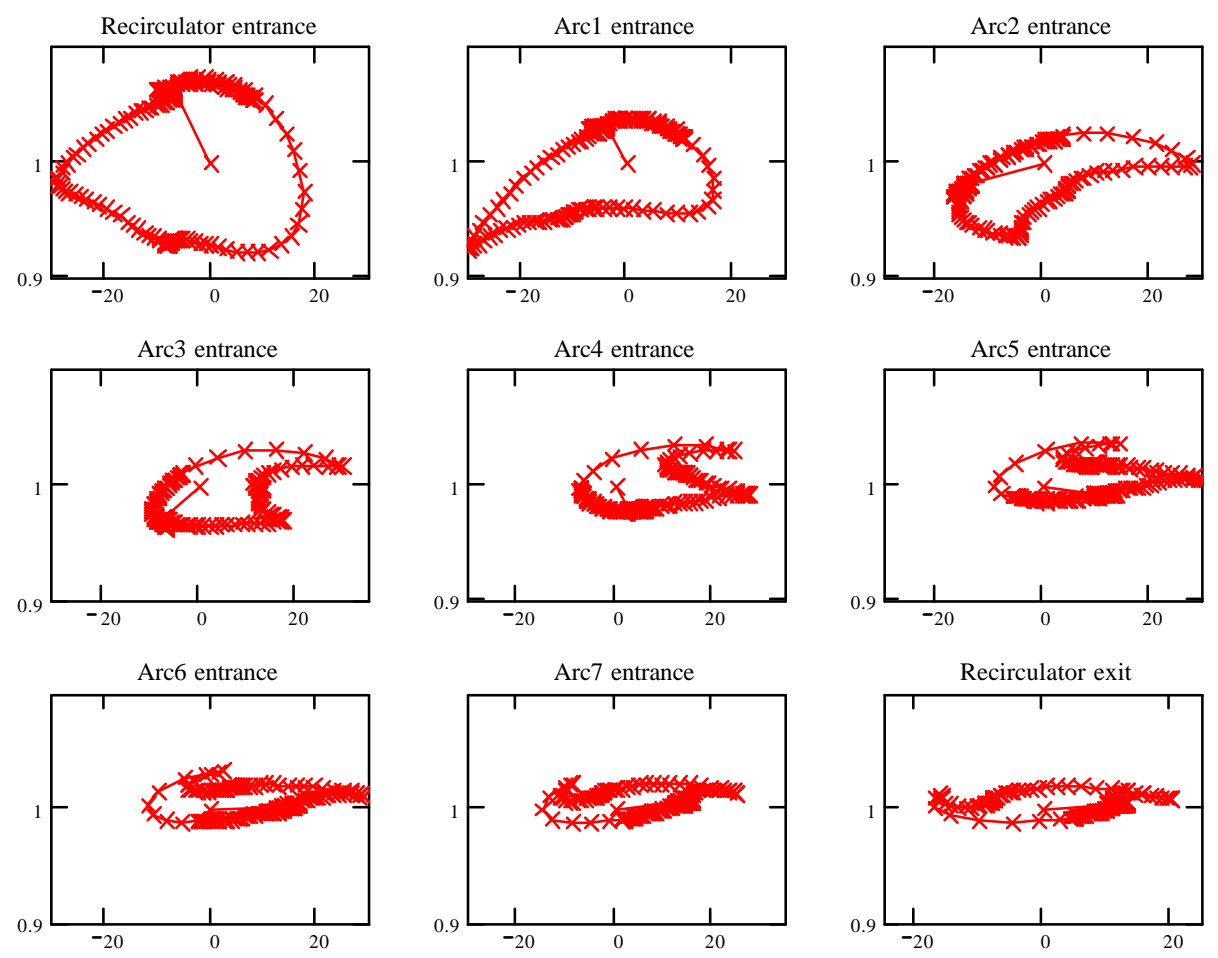

\section{Conclusions}

Results of this study suggest there are no obvious physical, or echnical limitations precluding construction of an RLA for acceleration of muons to $20 \mathrm{GeV}$.

The resulting optics is well suited for transporting large phase space beams. Proposed chromatic corrections via two families of sextupoles in spreaders/recombiners [3] are proven to be very effective in emittance dilution control.

\section{References}

[1] C.M. Ankenbrandt et al., "Status of Muon Collider Research and Development and Future Plans", Physical Review Special Topics - Accelerators and Beams, Vol 2, 081001 (1999)

[2] S.A. Bogacz and V.A. Lebedev, "Recirculating Linacs for Neutrino Factory - Arc Optics Design and Optimization", Nuclear Instruments and Methods in Physics Research A, Vol 472/3, 528, (2001)

[3] S.A. Bogacz and V.A. Lebedev, "Recirculating Linac Muon Accelerator for Neutrino Factory", Proceedings of NuFACT'01, May 2001 